\title{
Gating of the cAMP Signaling Cascade and Melatonin Synthesis by the Circadian Clock in Mammalian Retina
}

\author{
Chiaki Fukuhara, ${ }^{1}$ Cuimei Liu, ${ }^{1}$ Tamara N. Ivanova, ${ }^{2}$ Guy C.-K. Chan, ${ }^{3}$ Daniel R. Storm, ${ }^{3}$ P. Michael Iuvone, ${ }^{2}$ and \\ Gianluca Tosini ${ }^{1}$ \\ ${ }^{1}$ Neuroscience Institute and National Science Foundation Center for Behavioral Neuroscience, Morehouse School of Medicine, Atlanta, Georgia 30310- \\ 1495, ${ }^{2}$ Department of Pharmacology, Emory University School of Medicine, Atlanta, Georgia, 30322, and ${ }^{3}$ Department of Pharmacology, University of \\ Washington, Seattle, Washington, 98195
}

\begin{abstract}
Melatonin is synthesized in retinal photoreceptor cells and acts as a neuromodulator imparting photoperiodic information to the retina. The synthesis of melatonin is controlled by an ocular circadian clock and by light in a finely tuned mechanism that ensures that melatonin is synthesized and acts only at night in darkness. Here we report that the circadian clock gates melatonin synthesis in part by regulating the expression of the type 1 adenylyl cyclase (AC1) and the synthesis of cAMP in photoreceptor cells. This gating is effected through E-box-mediated transcriptional activation of the $\mathrm{AC} 1$ gene, which undergoes robust daily fluctuations that persist in constant illumination. The circadian control of the cAMP signaling cascade indicates that the clock has a more general and profound impact on retinal functions than previously thought. In addition, rhythmic control of $\mathrm{ACl}$ expression was observed in other parts of the central circadian axis, the suprachiasmatic nucleus and pineal gland, but not in other brain areas examined. Thus, clock control of the cAMP signaling cascade may play a central role in the integration of circadian signals that control physiology and behavior.
\end{abstract}

Key words: adenylyl cyclase; cAMP; retina; melatonin; circadian rhythm; AA-NAT; suprachiasmatic nucleus

\section{Introduction}

Circadian clocks are a conserved physiological feature of almost all organisms in which they play an important adaptive role. Retinal neurons of vertebrates, particularly photoreceptor cells, contain autonomous circadian clocks (Cahill and Besharse, 1993; Pierce et al., 1993; Tosini and Menaker 1996, 1998) that have been implicated in the regulation of several retinal functions from gene expression to visual sensitivity (for review, see Cahill and Besharse, 1995; Anderson and Green, 2000; Tosini and Fukuhara, 2002). Perhaps the most intensely investigated of these functions is melatonin biosynthesis, the synthesis of which in retinal photoreceptor cells displays a clear circadian rhythm in vivo and in vitro (Besharse and Iuvone, 1983; Cahill and Besharse 1993; Tosini and Menaker, 1996, 1998). Retinal melatonin is high at night and low during the day, and the changes in melatonin levels are a direct reflection of the change in the rate of transcription and activity of the enzyme arylalkylamine $N$-acetyltransferase (AA-NAT) (Iuvone et al., 1997; Niki et al., 1998; Sakamoto and Ishida, 1998; Fukuhara et al., 2001).

AA-NAT is a key regulatory enzyme in the melatonin biosyn-

Received Nov. 6, 2003; revised Dec. 17, 2003; accepted Jan. 1, 2004.

This work was supported by grants from the National Institutes of Health (NIH) (NS 43459), the National Science Foundation Center for Behavioral Neuroscience (IBN-9876754), and the NASA Cooperative Agreement NCC 9-58 with the National Space Biomedical Research Institute to G.T., and NIH grants to P.M.I. (EY04864) and D.R.S. (NS37056). We thank James Wessel III for the LCM and RNA extraction of the microdissected photoreceptor cells. Thanks also to Dr. D. C. Klein for comments and suggestions on a previous version of this manuscript.

Correspondence should be addressed to Dr. Gianluca Tosini, Neuroscience Institute, Morehouse School of Medicine, 720 Westview Drive Southwest, Atlanta, GA 30310-1495. E-mail: tosinig@msm.edu.

D01:10.1523/JNEUROSCI.4988-03.2004

Copyright $\odot 2004$ Society for Neuroscience $\quad$ 0270-6474/04/241803-09\$15.00/0 thetic pathway (Klein et al., 1997). In retina, AA-NAT is expressed primarily in photoreceptor cells (Bernard et al., 1997; Niki et al., 1998; Liu et al., 2004). The promoter region of the rat AA-NAT gene contains a cAMP-responsive element (CRE) (Baler et al., 1997) and an E-box (Chen and Baler, 2000). Thus, the transcription of this gene might be regulated by changes in cAMP levels, which in photoreceptor cells follow changes in illumination (for review, see Iuvone 1995), or directly by the circadian clock. Indeed, recent work has demonstrated that cAMP stimulates AA-NAT transcription and enzyme activity in photoreceptor cells (Iuvone et al., 1990; Greve et al., 1999; Chen and Baler, 2000; Ivanova and Iuvone, 2003). In addition, photoreceptor AA-NAT transcription is under direct control of the circadian clock via the action of the CLOCK:BMAL1 protein complex on the E-box contained in the proximal promoter of the gene (Chen and Baler, 2000; Tosini and Fukuhara, 2002). These dual mechanisms for regulating melatonin biosynthesis in the retina present an ideal model for investigating the interactions between changes in environmental light and the circadian clock.

In the present report, we investigated the time-of-daydependent effects of darkness, which increases photoreceptor cAMP levels (for review, see Iuvone, 1995), on retinal melatonin synthesis. Our results indicate the presence of a circadian mechanism that gates melatonin synthesis through transcriptional regulation of the type 1 adenylyl cyclase (AC1) by the BMAL1: CLOCK complex.

\section{Materials and Methods}

Animals and tissue collection. Adult male Sprague Dawley rats bred at Morehouse School of Medicine were maintained on a $12 \mathrm{hr}$ light/dark 
(LD) cycle of illumination with light on from Zeitgeber time (ZT) 0-12 for a least 1 week before experiments were performed. To investigate the expression in LD cycles, animals were killed at ZT 0, 4, 8, 12, 16, and 20. When animals were killed during the night, the procedure was performed in dim red light $(<0.1$ lux). To investigate the pattern of expression in constant conditions, rats were transferred into constant dim red light $(<0.1$ lux $)$ for $3 \mathrm{~d}$ before they were killed. In our experimental conditions, the free-running period was $\sim 24.1 \mathrm{hr}$ (our unpublished observation), and the phase shift on the fourth day was calculated to be negligible ( $<35 \mathrm{~min}$ ); therefore, no adjustment was made to the sampling schedule. Samples were collected on the fourth day at circadian time (CT) 0, 4, 8, 12,16 , and 20. Retinas were dissected, immediately frozen on dry ice, and stored at $-80^{\circ} \mathrm{C}$. For measurement of $\mathrm{AC} 1$ expression in the brain, animals were killed at ZT 8 and ZT 20, brains were removed and dissected, and the anatomical regions of interest were immediately frozen on dry ice and stored at $-80^{\circ} \mathrm{C}$.

Flow-through culture. Details about the flow-through culture technique and validation of melatonin radioimmunoassay are reported in previous studies (Tosini and Menaker, 1996, 1998). In brief, the eyes were removed from the animals under halothane anesthesia during the daytime. Neural retinas were isolated and placed in the flow-through apparatus. Retinas were then cultured at $33^{\circ} \mathrm{C}$ (flow rate $1 \mathrm{ml} / \mathrm{hr}$ ) in $\mathrm{LD}$ using medium 199 with Eagle's salts and L-glutamine supplemented with antibiotics (100 U/ml penicillin, $0.01 \mathrm{gm} / \mathrm{ml}$ streptomycin) and $10 \mu \mathrm{M}$ serotonin. On the second day of culture beginning at ZT $0,3,6,9$, or 12, retinas were exposed to darkness for $6 \mathrm{hr}$, and samples of perfusate were collected at $3 \mathrm{hr}$ intervals.

Melatonin assay. The amount of melatonin in the culture medium was measured by radioimmunoassay, using a modification (Tosini and Menaker, 1996, 1998) of the method of Rollag and Niswender (1976). The assay was validated with the following experiments. Melatonin $(0$, $10,25,50,100,200 \mathrm{pg}$ per tube; four replicates at each level) was added to pooled retinal perfusates collected at night. The exogenous melatonin was recovered quantitatively (linear regression $Y=0.95 \times+81.02 ; r=$ 0.99; $p<0.001 ; Y$-intercept, $81.02 \mathrm{pg}$, represents the endogenous concentration of melatonin in the sample of retinal perfusate). A dilution curve of retinal perfusate was constructed and found to be parallel to that of melatonin standards, indicative of equivalent binding of the melatonin antibody in unknowns and standards (ANOVA; $p>0.01$ ). The lower and upper limits of the radioimmunoassay sensitivity were 2.5 and $500 \mathrm{pg}$ per tube, respectively. In addition, we performed chloroform extractions of samples of medium collected during the day and during the night, and in both cases the extraction efficiency was $\sim 89 \%$. Because this procedure eliminates the only compounds other than melatonin known to have significant cross-reactivity with the antibody (Rollag and Niswender, 1976), this result makes it unlikely that we are measuring a substance other than melatonin. Because the procedure described above is not a complete validation, however, "melatonin" is used in this report as an abbreviation for "melatonin-like immunoreactivity."

cAMP measurement. Retinas were homogenized in 6\% TCA and centrifuged. Supernatant fractions were extracted repeatedly with ethyl ether, dried under nitrogen, and reconstituted in sodium acetate buffer, $\mathrm{pH}$ 6.2. cAMP levels were measured by radioimmunoassay (DuPont NEN, Boston, MA).

Real-time quantitative RT-PCR. Total RNA was extracted using Trizol (Life Technologies, Grand Island, NY) reagent after sonication. DNA was degraded by DNase I. First-strand cDNA was synthesized using Moloney murine leukemia virus reverse transcriptase and according to the manufacturer's protocol. Each set of samples was simultaneously processed for RNA extraction, DNase I treatment, cDNA synthesis, and PCR reaction. Real-time quantitative PCR was performed using an iCycler (Bio-Rad, Hercules, CA) in the presence of SYBR green I with primers to rat glyceraldehyde-3-phosphate dehydrogenase (GAPDH) (GenBank accession number X02231; 5'-AGACAGCCGCATCTTCTTGT-3', $5^{\prime}$-TGATGGAACAATGTCCACT-3') and rat adenylyl cyclase type I (AF053980; 5' -TAGCTCAAGGCTGTGTGGTG-3', 5' -ACTGAGCTCCAGGACAAGGA-3'). Amplification was performed after $30 \mathrm{sec}$ of denaturation at $95^{\circ} \mathrm{C}$. Once the temperature reached $95^{\circ} \mathrm{C}$, it was decreased to $60^{\circ} \mathrm{C}$, maintained for $20 \mathrm{sec}$, and raised to $72^{\circ} \mathrm{C}$ for 30 sec. Fluorescence was measured at the melting temperature after each cycle, allowing comparison of fluorescence intensities among samples while their increase is within the linear range. Quantification of AC1 and GAPDH cDNAs was accomplished by comparing the threshold cycles for amplification of the unknowns with those of four concentrations of $\mathrm{ACl}$ and $\mathrm{GAPDH}$ standards using the $i$ Cycler software. Melting temperatures used were as follows: GAPDH, $86^{\circ} \mathrm{C}$; $\mathrm{AC} 1$, $89^{\circ} \mathrm{C}$. After 40 cycles of reactions, the PCR products were run on an agarose gel to verify that a single amplicon of appropriate size was amplified. AC1 mRNA levels were normalized using GAPDH mRNA levels. In each experimental set, the maximum value was set to 100 , and each value was normalized relative to that maximum value (Fukuhara et al., 2002).

Laser capture microdissection of photoreceptor cells. Whole eyes were embedded in OCT (Tissue-Tek, Torrance, CA), frozen on dry ice, and stored at $-80^{\circ} \mathrm{C}$. Frozen tissues were cut at $8 \mu \mathrm{m}$ thickness and mounted on uncharged glass slides (VWR Scientific). The frozen sections were thawed for $30 \mathrm{sec}$ and fixed in $75 \%$ ethanol for $30 \mathrm{sec}$ followed by a wash in RNase-free water for $30 \mathrm{sec}$. The sections were stained with HistoGene (Arcturus Engineering, Mountain View, CA) staining solutions for $15 \mathrm{sec}$ followed by a wash with RNase-free water for $30 \mathrm{sec}$. The sections were dehydrated in graded ethanol solutions (75\%, $30 \mathrm{sec} ; 95 \%$, $30 \mathrm{sec} ; 100 \%$, $30 \mathrm{sec}$ ) and cleared in xylene (5 min). After air-drying for $30 \mathrm{~min}$, the slides were kept in a vacuum desiccator for a minimum of $2 \mathrm{hr}$. Laser capture was performed by lifting the outer nuclear layer and inner segments of photoreceptor cells onto HS-CapSure non-contact laser capture microdissection (LCM) film using a PixCell IIe LCM system (Arcturus Engineering). Total RNA was extracted from the captured cells using the PicoPure RNA Isolation Kit (Arcturus Engineering). Samples were reverse transcribed and subjected to real-time PCR analysis as described above.

Immunocytochemistry. Eyes, obtained from animals killed at ZT8 and ZT 20, were punctured and then fixed with $4 \%$ paraformaldehyde in PBS, $\mathrm{pH} 7.4$, for $6 \mathrm{hr}$ at $4^{\circ} \mathrm{C}$. The eyes were then transferred to a solution of $30 \%$ sucrose for $24 \mathrm{hr}$ at $4^{\circ} \mathrm{C}$, embedded in Tissue-Tek OCT compound (Miles, Elkhart, IN), and cut into 15- to 20 - $\mu \mathrm{m}$-thick cryosections. Sections were then mounted on SuperFrost/plus slides (Fisher Scientific, Houston, TX). After three 10 min washes in PBS and 30 min incubation at room temperature in PBS containing $0.6 \% \mathrm{H}_{2} \mathrm{O}_{2}$ to block endogenous peroxidase activity, the sections were incubated in PBS containing $10 \%$ goat serum and $0.3 \%$ Triton $\mathrm{X}-100$ for $20 \mathrm{~min}$ at room temperature to reduce nonspecific binding. Subsequently, the section were incubated for $45 \mathrm{~min}$ at room temperature followed by overnight incubation at $4^{\circ} \mathrm{C}$ in PBS containing $2 \%$ goat serum and $2 \mu \mathrm{g} / \mathrm{ml}$ of the rabbit polyclonal affinity-purified antibody for AC1 (sc-586, polyclonal antibody; Santa Cruz Biotechnology, Santa Cruz, CA). This amount of primary antibody $(2 \mu \mathrm{g} / \mathrm{ml})$ corresponds to a 1:50 dilution of the antibody solution provided. Sections were washed three times in PBS and incubated for $1 \mathrm{hr}$ with the biotinylated secondary antibody (goat anti-rabbit IgG) at a 1:500 dilution in PBS containing $1 \%$ goat serum. The sections were washed (three times) for $10 \mathrm{~min}$ in PBS before the detection of immunolabeling with avidin-biotin-peroxidase, with $\mathrm{H}_{2} \mathrm{O}_{2}$ and 3,3'-diaminobenzidine as substrate and nickel ammonium sulfate intensification (Vector Laboratories, Burlingame, CA). To determine the specificity of the antibody, negative controls were performed by omitting the primary antibody or by preabsorption with the blocking peptide (sc-586P) provided by Santa Cruz Biotechnology. Slides were coverslipped and viewed using a Zeiss Axioskop microscope.

Retinal cell culture. Isolated retinas were placed in DMEM and treated for $5 \mathrm{~min}$ in $0.025 \%$ trypsin with DNase (Worthington Biochemical Corporation, Lakewood, NJ) at $37^{\circ} \mathrm{C}$. Retinas were triturated with glass fire-polished pipettes and added to an excess of culture medium consisting of DMEM with glutamine, 10\% newborn calf serum (NCS), and antibiotics (100 U/ml penicillin, $100 \mathrm{~g} / \mathrm{ml}$ streptomycin). The cells were centrifuged at $300 \times g$ for $4 \mathrm{~min}$ and then resuspended in fresh DMEM medium. Cells were plated into polylysine-coated 12 -well plates (Becton Dickinson, Bedford, MA) at a density of $7 \times 10^{5}$ cells per well and supplemented with $0.8 \mathrm{ml}$ of DMEM/8\% NCS.

Transfection and luciferase reporter gene assay. On the second day of 

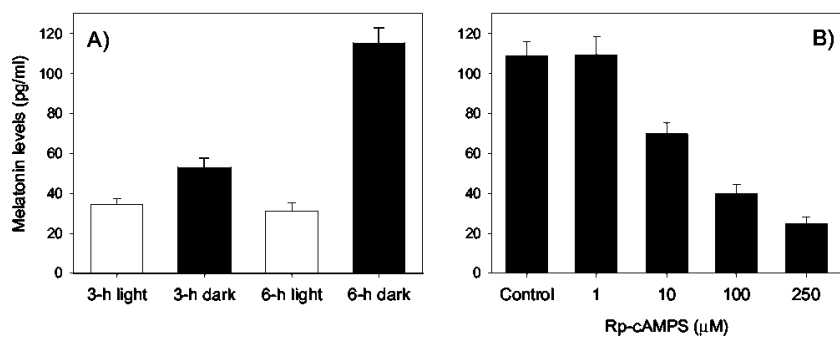

Figure 1. A, Effect of darkness in the middle of the day on melatonin levels. Retinas were cultured in the flow-through apparatus for $1 \mathrm{~d}$ on an LD cycle; on the second day, the retinas were exposed to complete darkness for $3-6 \mathrm{hr}$ beginning at ZT 6 . Melatonin levels began to increase after $3 \mathrm{hr}$ of darkness and reached nighttime levels after $6 \mathrm{hr}$ ( $t$ test; $p<0.01$ ). $B$, Rp-CAMPS (a PKA type Il inhibitor) inhibits dark-dependent melatonin synthesis. Medium 199 was supplemented with Rp-CAMPS $3 \mathrm{hr}$ before exposure to darkness. The control and all drugtreated groups were incubated in darkness for $6 \mathrm{hr}$ beginning at ZT 6. Rp-CAMPS inhibited the dark-induced melatonin synthesis in a dose-dependent manner $(p<0.001)$. Data are expressed as mean \pm SEM in cultured retinas ( $n=5$ in all groups).

culture, the NCS was reduced to $1 \%$ for subsequent transfection. Effectene Transfection Reagent (Qiagen, Valencia, CA) with $0.3 \mu \mathrm{g}$ DNA and $2.4 \mu \mathrm{l}$ enhancer was added to each well of the 12-well plate (Fukuhara et al., 2002; Tosini and Fukuhara, 2002). AC1 promoter-luciferase construct or AC1 Emut promoter-luciferase cloned in the pGL3-Basic Vector (Promega, Madison, WI) was cotransfected with either BMAL1 and CLOCK expression vectors (cloned in pCl-neo mammalian expression vector; Promega, Madison, WI) or empty vector. Cells were also cotransfected with Renilla luciferase(Promega), an internal control for transfection efficiency. Cells were harvested $24 \mathrm{hr}$ after transfection. Mutation at the E-box was introduced using the QuickChange site-directed mutagenesis kit (Stratagene, Cedar Creek, TX) with the following primers: 5'-CGCCCAAGGGGCCAGTGACCTTGGGCACC-3' and 5' -GGTGCCCAAGGTCACTGGCCCCTTGGGCG-3' . Details about AC1 promoter constructs have been described in a previous study (Chan et al., 2001). Increased cAMP levels reduce AC1 promoter-luciferase activity (Chan et al., 2001), indicating a negative feedback regulation of promoter activity. To inhibit the increase of cAMP levels, cells were treated with the adenylyl cyclase inhibitor SQ-22,536 (200 $\mu \mathrm{M})$ for $6 \mathrm{hr}$ before harvest. At least three wells were used to obtain $n=1$, and at least three independent experiments were performed. Luciferase assay was performed using the Dual-Luciferase Assay System (Promega) and a Berthold luminometer by following manufacturers' protocols. Each Firefly relative luciferase unit (RLU) was normalized to the Renilla RLU and the mean value of the $\mathrm{AC} 1+$ neo group set to 1 .

\section{Results}

\section{Melatonin synthesis in the retina is gated by the} circadian clock

Rat retinas were cultured in a flow-through apparatus in a $12 \mathrm{hr}$ LD cycle. During the second day of culture, retinas were exposed to a $6 \mathrm{hr}$ period of darkness beginning in the middle of the day (ZT 6). Melatonin levels began to increase $3 \mathrm{hr}$ after the start of the dark exposure and reached a maximum after $6 \mathrm{hr}$ (Fig. 1A). Melatonin levels after $6 \mathrm{hr}$ of darkness were not different from the levels measured in the middle of the night ( $t$ test; $p>0.1$; data not shown). Previous studies have shown that induction of melatonin synthesis depends on activation of the cAMP signaling pathway, which stimulates the activity of the melatonin-synthesizing enzyme AA-NAT by transcriptional and posttranslational mechanisms (Iuvone et al., 1997; Klein et al., 1997; Klein et al., 2002). Indeed, when retinas were exposed to $6 \mathrm{hr}$ of darkness with the culture medium containing different concentrations of RpcAMPS (a membrane-permeable protein kinase A (PKA) type II inhibitor), melatonin synthesis was inhibited in a dosedependent manner (Fig. $1 B)(p<0.001)$.
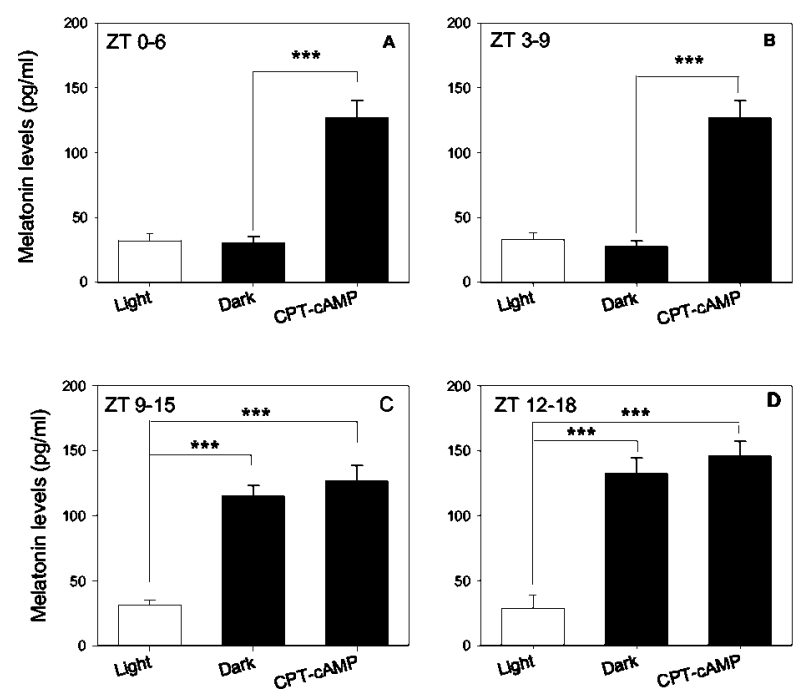

Figure 2. Effect of $6 \mathrm{hr}$ darkness or $100 \mu \mathrm{m} 8 \mathrm{p}$ CTP-CAMP beginning at ZT $0(A), 3(B), 9$ ( $C$, and $12(D)$ on melatonin levels \pm SEM in cultured retinas ( $n=5$ in all groups). Darkness induced melatonin synthesis at ZT 9 and 12 , but not at ZT 0 and 3.8 CTP-CAMP induced melatonin synthesis at all times. Data are expressed as mean \pm SEM in cultured retinas $(n=5$ in all groups). ${ }^{* *} p<0.001$

The ability to stimulate retinal melatonin biosynthesis with darkness is dependent on the time of day that the stimulus is applied. When retinas were exposed to darkness for $6 \mathrm{hr}$ at different times of the day, induction of melatonin synthesis was observed with incubations ending at ZT 12,15 , and 18, but not at ZT 6 or 9 (Fig. 1 A, 2), demonstrating that the induction of melatonin synthesis in darkness is gated by the circadian clock.

\section{Adenylyl cyclase serves as a gate for melatonin synthesis}

When the retinas were exposed to $6 \mathrm{hr}$ of darkness in the presence of $100 \mu \mathrm{M}$ of $8 \mathrm{p}$-CPT-cAMP (a membrane-permeable cAMP analog), melatonin release was significantly increased at all times tested (ZT 6 to ZT 18; $p<0.01$ in all cases) (Fig. 2). The differential time-of-day-specific effects of darkness and $8 \mathrm{p}-\mathrm{CPT}$ cAMP demonstrate that the mechanism(s) gating the synthesis of melatonin during the day is located upstream of PKA, possibly at the level of adenylyl cyclase. We decided, therefore, to determine whether forskolin (a direct activator of adenylyl cyclase) could induce melatonin synthesis in the morning at a time that was refractory to darkness but not to 8p-CPT-cAMP. Exposing cultured retinas to darkness from ZT 3 to ZT 9 in the presence of forskolin $(50 \mu \mathrm{M})$ and 3-isobutyl-1-methylthanine (IBMX) (100 $\mu \mathrm{M})$ failed to produce a significant change in melatonin levels (Fig. 3). The ability of 8pCPT-cAMP, but not darkness and forskolin, to stimulate melatonin formation in the morning suggests that at this time of day AC may be underexpressed or inhibited relative to later in the day and that $\mathrm{AC}$ serves as a gate for melatonin synthesis.

In retinas exposed to light, $8 \mathrm{pCPT}$-cAMP increased melatonin synthesis at all times of day tested (Fig. 4). In contrast, forskolin $(50 \mu \mathrm{M})$ or a combination of forskolin and IBMX $(100 \mu \mathrm{M})$ induced melatonin synthesis in light-exposed retinas only at ZT 6-12 and ZT 9-15 (Fig. 4). IBMX alone had no effect on melatonin synthesis of light-exposed retinas at any time of day, consistent with the known suppression of photoreceptor cAMP synthesis by light. 


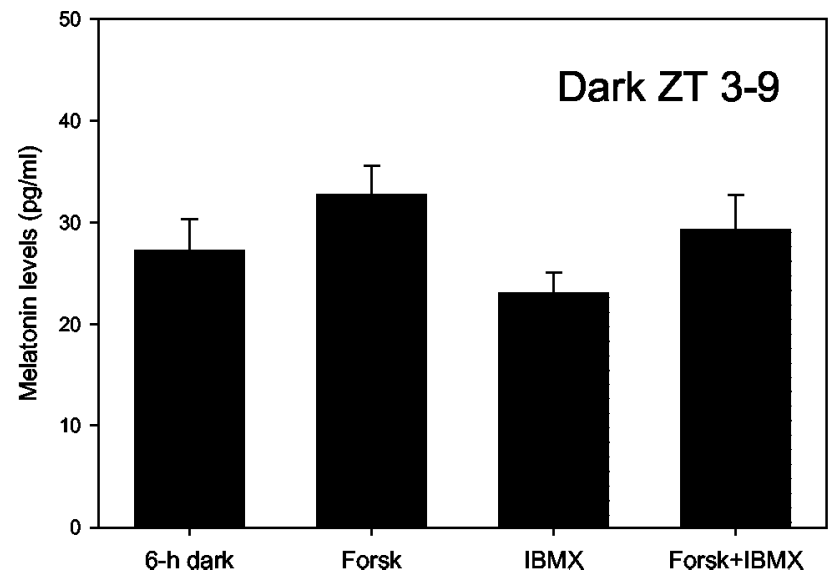

Figure 3. Lack of effect of forskolin and darkness in the morning on melatonin synthesis. Retinas were cultured in darkness from ZT 3 to ZT 9 in the presence of the adenylyl cyclase activator forskolin $(50 \mu \mathrm{M})$ and the cyclic nucleotide phosphodiesterase inhibitor IBMX (100 $\mu \mathrm{M}) . n=5$ per group. Neither forskolin nor IBMX, alone or in combination, stimulated melatonin synthesis in the morning $(p>0.05)$.
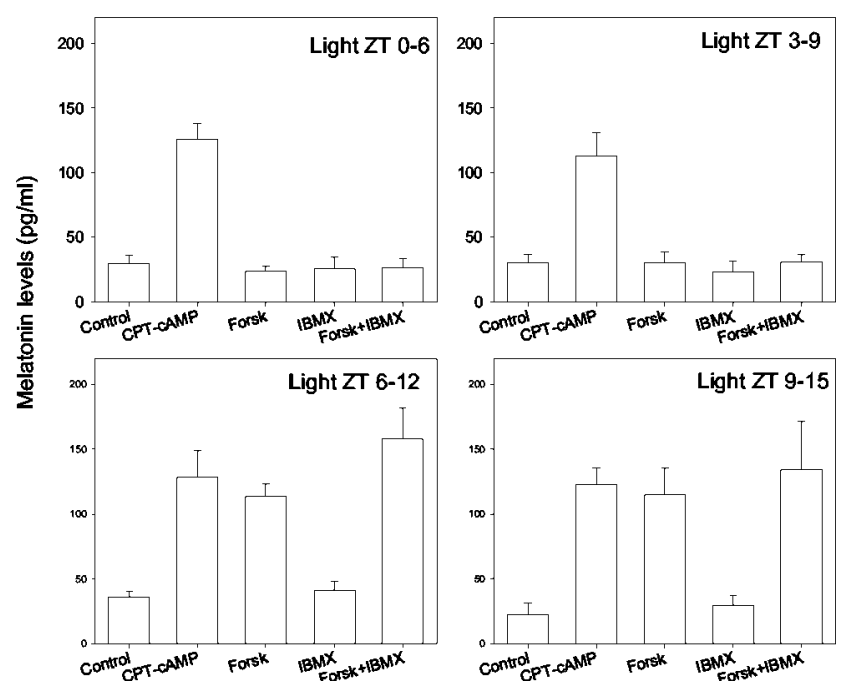

Figure 4. Effect of $6 \mathrm{hr}$ light, light plus $100 \mu \mathrm{m} 8 \mathrm{pCPT}-\mathrm{cAMP}$, light plus forskolin $(50 \mu \mathrm{M})$, light plus IBMX $(100 \mu \mathrm{M})$, and light plus forskolin $(50 \mu \mathrm{M})$ with IBMX (100 $\mu \mathrm{M})$ on cultured retinas. $n=4$ per group. Neither forskolin nor IBMX, alone or in combination, stimulated melatonin synthesis in the morning at ZT $0-6$ or at ZT $3-6$. Forskolin alone increased melatonin synthesis at ZT $6-12$ and ZT $9-15(p<0.01)$, and 8pCPT-CAMP increased melatonin synthesis in the light at all times examined $(p<0.01)$.

\section{Adenylyl cyclase transcription and activity are under circadian control}

There are several different forms of adenylyl cyclase in mammalian tissues (Xia and Storm, 1997). One of these, AC1, is neural specific, activated by $\mathrm{Ca}^{2+}$ and calmodulin (CaM), and highly expressed in photoreceptor cells (Xia et al., 1993). The induction of retinal AA-NAT by darkness is $\mathrm{Ca}^{2+}$ dependent, requires $\mathrm{Ca}^{2+}$ influx through voltage-gated channels, and is stimulated by cAMP (Iuvone and Besharse, 1986a,b). These observations suggest that a $\mathrm{Ca}^{2+} / \mathrm{CaM}$-stimulated cyclase may play a critical role in AA-NAT regulation. Therefore, we investigated the expression pattern of $A C 1$ in the rat retina in $L D$ and constant dim light using quantitative real-time RT-PCR. AC1 mRNA levels showed a significant rhythm in both conditions (Kruskal-Wallis test; $p<$ 0.05 in both cases). The mRNA peaked during the day, and the lowest levels were observed in the late night to early morning (Fig.
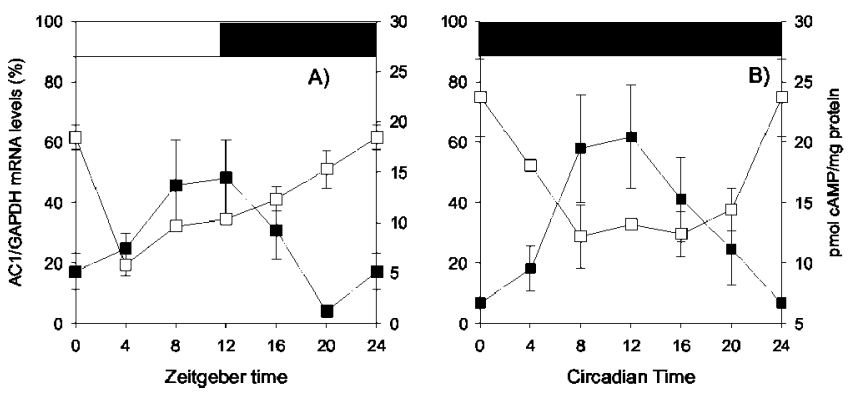

Figure 5. $A C 1 \mathrm{mRNA}$ and CAMP levels in rat retina. $A$, Retinal samples were collected under $\mathrm{LD}$ cycles at the times indicated. $B$, Animals were first entrained to the $\mathrm{LD}$ cycle and then transferred to constant dim red light ( $<0.1$ lux) for $3 \mathrm{~d}$ before they were killed. The open bar at the top of the graph represents the period of light; the closed bars represent period of darkness $(A)$ or dim red light $(B)$. AC1 mRNA (black squares) and CAMP (white squares) levels were measured using real-time quantitative RT-PCR and radioimmunoassay, respectively. AC1 mRNA abundance was rhythmic in $\mathrm{LD}(p=0.037)$ and constant dim light $(p=0.028)$. cAMP levels were rhythmic in LD $(p<0.001)$ and in constant dim light $(p=0.011)$. Data are expressed as mean \pm SEM in cultured retinas ( $n \geq 5$ retinas per group for AC1 mRNA measurements; $n=3$ retinas per group for (AMP measurements).
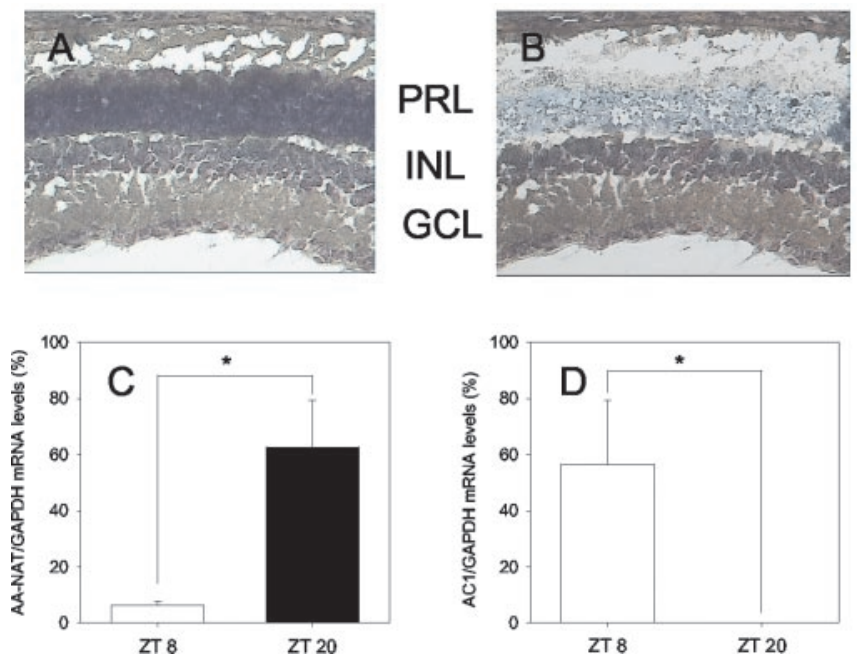

Figure 6. Laser capture microdissection of individual cell layers of rat retina. Photomicrographs of a retinal section before $(A)$ and after $(B)$ the photoreceptors layer has been serially captured using LCM. Aa-nat and AC1 transcript levels in microdissected photoreceptor cells. Photoreceptor somata and inner segments were collected by LCM from $8 \mu \mathrm{m}$ frozen sections of retinas obtained at ZT 8 and ZT 20. Total RNA was extracted from the captured material and subjected to real-time RT-PCR for GAPDH, Aa-nat ( $C$, and $A C 1(D) . n=4$ per group. Aa-nat mRNA was significantly higher at ZT 20 than at ZT $8(p=0.029)$. AC1 transcript level was significantly higher at ZT 8 than at ZT 20 ( $p=0.027$; Mann-Whitney rank sum test).

$5 A)$. The changes in mRNA levels were reflected by a rhythm of cAMP content (Fig. 5B), demonstrating that the activity of adenylyl cyclase is rhythmic in situ. In LD, cAMP levels were lowest in the midmorning, when darkness and forskolin have no effect on melatonin production; levels of the cyclic nucleotide increase progressively through the day, peaking in the late night. This rhythm of cAMP level is delayed by several hours relative to the rhythm of AC1 mRNA, which is consistent with the time needed for synthesis and processing of the membrane protein (Tzavara et al., 1996).

To determine whether the rhythm of $A C 1$ mRNA level occurs in photoreceptor cells, we examined the expression of mRNAs encoding $A C 1$ and $A a$-nat in photoreceptor somata and inner segments isolated by laser capture microdissection (Fig. 6). Aanat mRNA levels in photoreceptor cells were low during the day- 

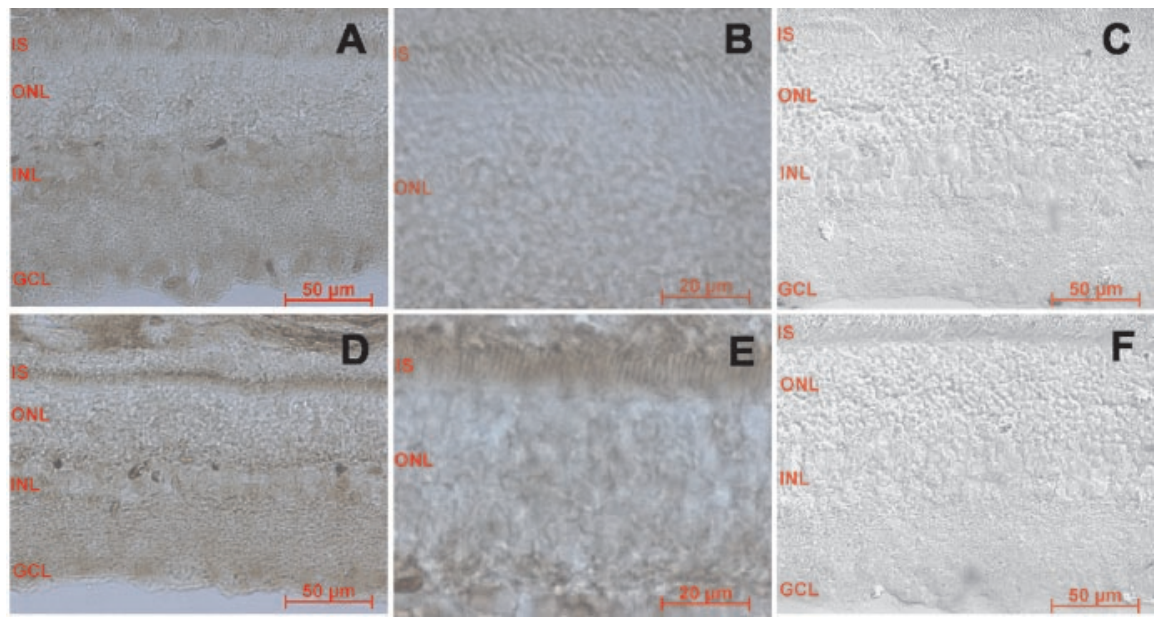

Figure 7. Immunohistochemical localization of $A C 1$ in the rat retina at ZT $8(A-C)$ and ZT $20(D-F)$. Retinal sections were processed for immunohistochemistry using $A C 1$ affinity-purified antibody. $B, E$, High-magnification views of the photoreceptor layer. IS, Inner segment; $0 \mathrm{NL}$, outer nuclear layer; INL, inner nuclear layer; $\mathrm{GCL}$, ganglion cell layer. Micrographs are representative of results obtained from at least four animals at each time point. C, F, Controls (preabsorption with the blocking peptide).

transcriptional competence of the $\mathrm{AC} 1$ E-box, a rat AC1 promoter-luciferase reporter construct was transfected into cultured retinal cells with either empty (neo-) or BMAL1 and CLOCK expression vectors. As shown in Figure 8, AC1 promoterluciferase activity was significantly increased by cotransfection of BMAL1 and CLOCK. More significantly, mutation of the E-box within the AC1 promoterluciferase reporter construct completely blocked reporter gene expression in retinal cells even when cotransfected with BMAL1 and CLOCK.

\section{AC1 mRNA expression in other parts of the brain}

AC1 is neural specific and expressed only in specific areas of the brain such as neocortex, cerebellum, olfactory bulb, hippocampus, and pineal gland (Xia et al., 1993). Therefore, we questioned whether

time (ZT 8) and high at night (ZT 20), consistent with previous measurements on whole rat retina (Sakamoto et al., 1998, 2002). In contrast, the AC1 transcript level in photoreceptor cells was high at ZT 8 and essentially undetectable at ZT 20. This result demonstrates that the $A C 1$ and $A a$-nat genes are both rhythmically expressed in photoreceptor cells and supports a causal relationship between the circadian control of $\mathrm{ACl}$ and the gating of melatonin biosynthesis.

\section{Rhythmic AC1 immunoreactivity in the photoreceptors}

A previous paper reported that AC1 immunoreactivity is not present in rat photoreceptors (Abdel-Majid et al., 2002); however, that study analyzed AC1 immunoreactivity during the daytime. We decided therefore to determine whether AC1 immunoreactivity varies with the time of day. Two time points (ZT 8 and ZT 20) were selected for analysis on the basis of the observation that cAMP levels are low at ZT 8 and high at ZT 20 (Fig. 5). In addition, a previous study has described a long temporal delay between the peaks of $\mathrm{AC} 1$ transcript level and $\mathrm{Ca}^{2+}$ /calmodulinstimulated adenylyl cyclase activity in rat pineal (Tzavara et al., 1996); transcript levels were high at midday and enzyme activity was high at night. Thus, on the basis of our measurements of AC1 mRNA levels in retina, we predicted that AC1 protein levels would be high at ZT 20 and low at ZT 8. Photoreceptor inner segments showed strong AC1 immunoreactivity at ZT 20 (Fig. $7 D, E$ ) but not at ZT 8 (Fig. $7 A, B$ ). This labeling was abolished by preabsorption of the primary antibody with blocking peptide (Fig. 7C,F). AC1 immunoreactivity was also present in the inner nuclear layer and in the ganglion cell layer, but no difference in immunoreactivity between the two time points was observed (Fig. $7 A, B$ and $D, E$ ).

\section{AC1 transcription is controlled by BMAL1:CLOCK}

Several lines of evidence indicate that E-boxes are an integral part of the cis-acting element driving the circadian transcription of clock-controlled genes (Jin et al., 1999; Chong et al., 2000; Ripperger et al., 2000). A previous study identified an E-box (CACGTG) in the promoter region of the $\mathrm{AC} 1$ gene (Chan et al., 2001), indicating that the circadian oscillator might directly control the transcription of this gene by the action of the BMAL1: CLOCK complex, which trans-activates E-boxes. To assess the
$A C 1$ transcription is under direct control of the circadian clock in these tissues, as well as in retina. As shown in Figure 9A-C, AC1 mRNA levels during the daytime (ZT 8) were not significantly different from those at night (ZT 20) in the hippocampus, cerebellum, and olfactory bulb. In contrast, $A C 1$ transcript levels in the pineal gland and suprachiasmatic nucleus (SCN) show clear daily rhythms $(p<0.05$ and $p<0.01$ ) (Fig. 9D,E). Because our study investigated AC1 mRNA at only two time points, no definitive conclusion can be reached regarding the absence of rhythmicity in neocortex, cerebellum, olfactory bulb, and hippocampus.

\section{Discussion}

Retinal melatonin levels are regulated by the interaction between the circadian clock and the photic environment. Several studies have demonstrated that the rhythm in melatonin release from cultured retinas free-runs in constant darkness, is entrainable by the light, and is temperature compensated (Tosini and Menaker, 1996, 1998). The fact that this rhythm shows the three fundamental characteristics of a circadian clock (i.e., free-run, entrainment, and temperature compensation) demonstrates that melatonin synthesis in the retina is controlled by a bona fide circadian clock located within this tissue. In addition to clock control, retinal melatonin levels rapidly (5-10 min) decrease after light exposure at night (Hamm et al., 1983; Fukuhara et al., 2001) to ensure that melatonin is released and acts only in darkness. As in the pineal gland (Gastel et al., 1998), the rapid reduction in melatonin levels is achieved by a rapid decrease in AA-NAT enzyme by proteasomal proteolysis (Fukuhara et al., 2001; Iuvone et al., 2002).

The results presented here, as well as those of previous studies, have led to the following working hypothesis on the control of AA-NAT activity in photoreceptor cells by the circadian clock and light (Fig. 10). The circadian clock directly controls the rhythmic expression of Aa-nat mRNA through the BMAL1: CLOCK complex and its interaction with the E-box located on the Aa-nat promoter. The circadian clock also provides for the rhythmic expression of AC1, temporally regulating the ability of $\mathrm{Ca}^{2+}$ to stimulate cAMP synthesis. Darkness depolarizes the photoreceptor plasma membrane, leading to $\mathrm{Ca}^{2+}$ influx and stimulation of cAMP synthesis late in the day and at night, when $\mathrm{AC} 1$ protein expression is high, but not during the early morning. 


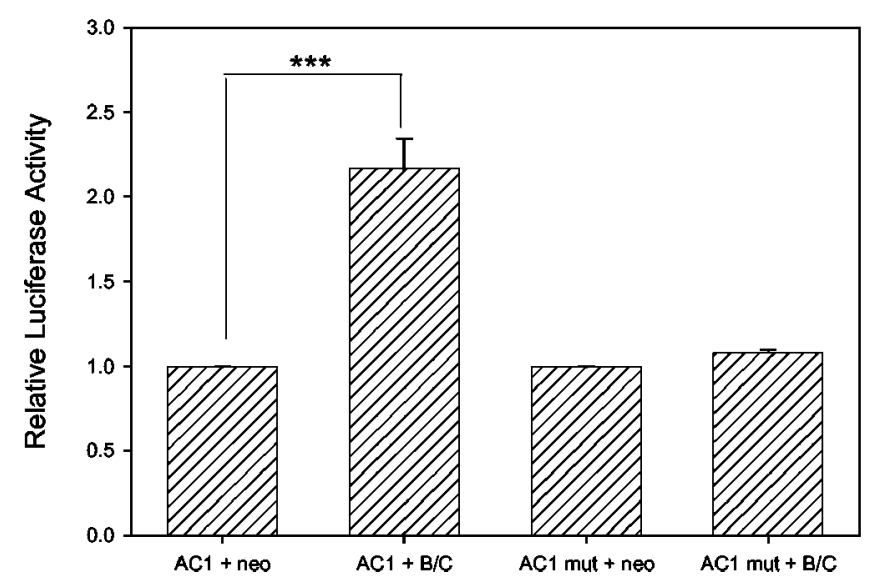

Figure 8. AC1 transcriptional regulation by BMAL1 and CLOCK. AC1 promoter-driven luciferase activity in retinal cultures was significantly increased by cotransfection of BMAL1 and CLOCK with respect to the activity obtained by cotransfecting with empty expression vector $(t$ test; $p<0.01$ ). AC1 mut luciferase construct (i.e., a construct in which the E-box has been mutated from CACGTG to CACTG) was not activated by cotransfecting with BMAL1 and CLOCK or with empty expression vector ( $t$ test; $p>0.1)$. Values are means \pm SEM $(n=3$ for each of the three independent experiments).
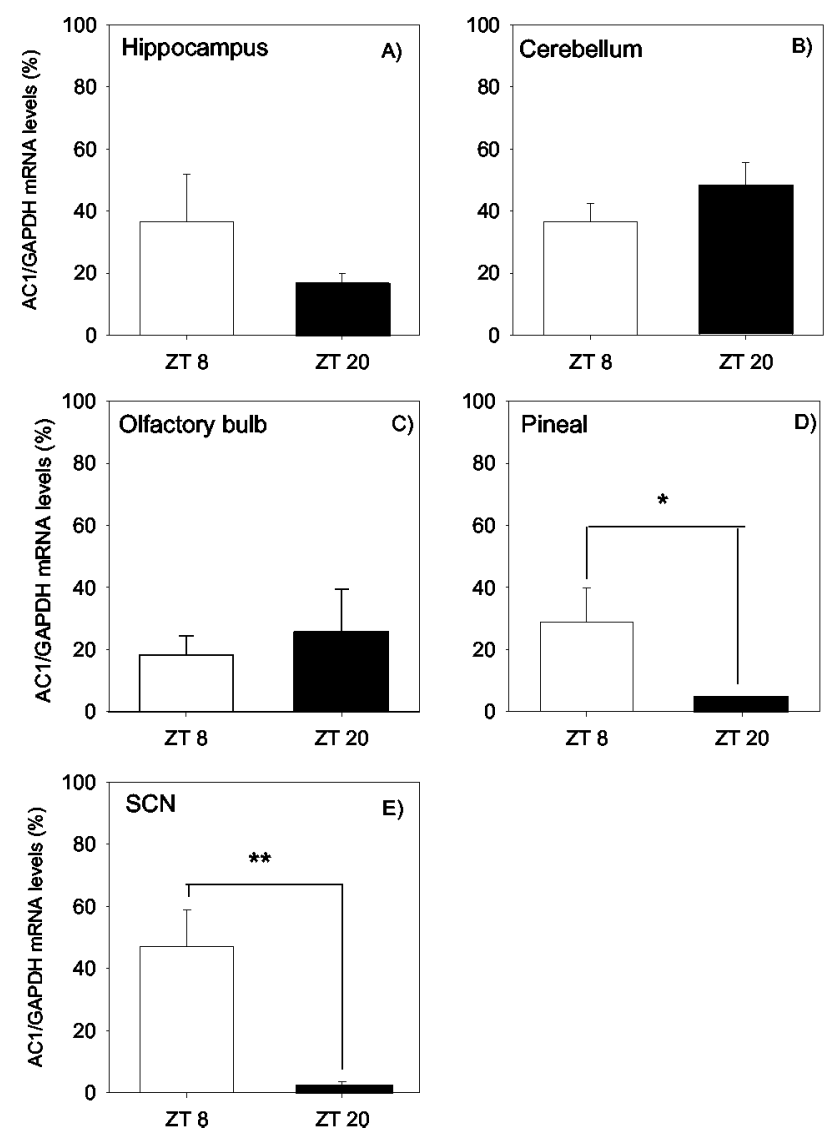

Figure 9. Daily variation of $A C 1$ expression in the hippocampus $(A)$, cerebellum $(B)$, olfactory bulb $(C)$, pineal $(D)$, and SCN $(E)$ measured using real-time quantitative RT-PCR. In the pineal and in the SCN, AC1 mRNAs were significantly higher at ZT 8 than at ZT 20. Data are expressed in mean (SEM; $n \geq 8)$. ${ }^{* *} p<0.01 ;{ }^{*} p<0.05$.

Thus, AC1 expression provides a gate controlling the time of day when cAMP levels are maximally stimulated by darkness. The increase of cAMP level in darkness stimulates $A a$-nat transcription via PKA and the CRE present in the Aa-nat promoter, in-
Circadian Clock

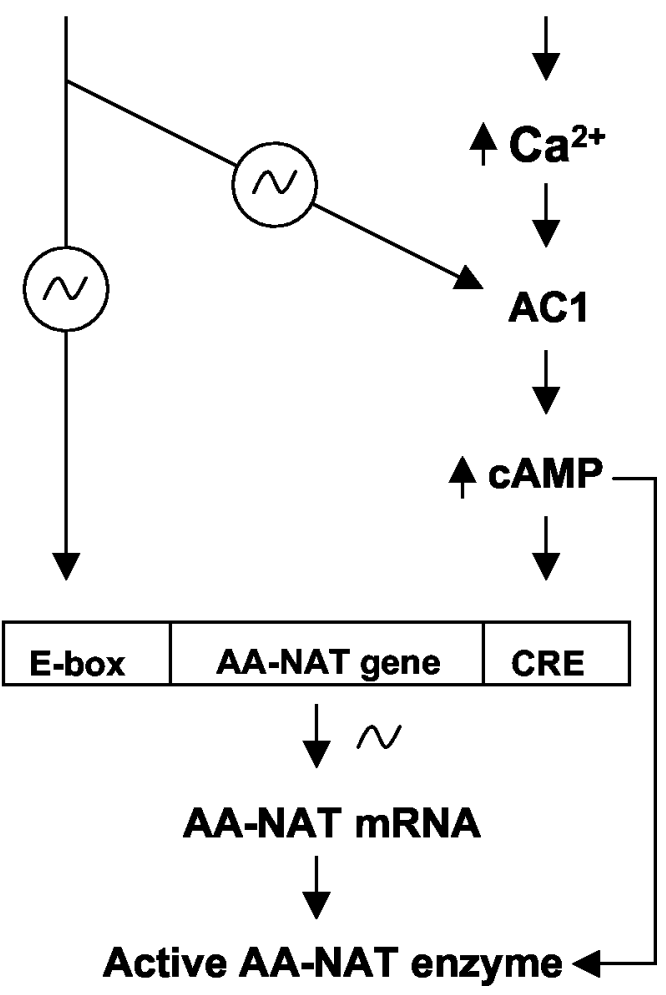

Figure 10. Schematic diagram illustrating regulation of melatonin synthesis by the circadian clock and darkness in photoreceptor. See Discussion for details.

creasing the rate of AA-NAT protein synthesis. In addition, the increase of cAMP and PKA activity phosphorylates and stabilizes AA-NAT, leading to its interaction with 14-3-3 proteins and inhibition of its degradation by proteasomes. Thus, melatonin synthesis is stimulated by darkness late in the day and at night, but not during the morning. Light exposure at night, which rapidly decreases melatonin synthesis, does so by hyperpolarizing the photoreceptor cell, leading to decreased intracellular $\mathrm{Ca}^{2+}$ and cAMP levels and the dephosphorylation and degradation of AANAT. Although some aspects of this hypothesis remain untested, it is consistent with the present data and an extensive literature.

The effects of daytime exposure to darkness on retinal melatonin synthesis and release have been investigated, but the results obtained are contradictory. Redburn and Mitchell (1989) reported that exposure to darkness during the day induced (within $1 \mathrm{hr}$ ) rapid synthesis and releases of melatonin from the retina, whereas Faillace et al. (1994) found melatonin levels increased only after a much longer period $(6 \mathrm{hr})$ of darkness. In the present study we investigated the effect of daytime exposure to darkness on melatonin synthesis and release in the rat retina at different times of the day. Our data demonstrate that exposing cultured rat retina to darkness can only activate melatonin synthesis in the middle to late day and at night (Fig. 2). Darkness early in the day has no effect. The stimulation of melatonin synthesis that occurs in darkness is attributable to the activation of cAMP signaling cascade (Fig. $1 \mathrm{~B}$ ), as it is in retinas of non-mammalian vertebrates (Iuvone and Besharse, 1983, 1986a,b; Hasegawa and Cahill, 2000).

The signaling pathway responsible for the activation of melatonin synthesis in chick retina has been partially elucidated (for review, see Iuvone et al., 1997, 1999). In this experimental model, depolarization of photoreceptors, as happens in darkness (Wer- 
blin and Dowling, 1969), induces AA-NAT activity by a $\mathrm{Ca}^{2+}$ and cAMP-dependent mechanism (Avendano et al., 1990; Gan et al., 1995; Ivanova and Iuvone, 2003). Depolarization of the photoreceptor membrane opens dihydropyridine-sensitive voltagegated $\mathrm{Ca}^{2+}$ channels, resulting in a large, sustained increase in intracellular $\mathrm{Ca}^{2+}$ concentration in photoreceptor inner segments and the $\mathrm{Ca}^{2+}$-dependent stimulation of cAMP formation, apparently by activating calmodulin-stimulated adenylyl cyclase (Iuvone et al., 1991; Gan et al., 1995; Uchida and Iuvone, 1999). The increase of intracellular cAMP activates AA-NAT gene transcription and decreases AA-NAT protein degradation, consequently stimulating AA-NAT activity and melatonin synthesis (Baler et al., 1997; Alonso-Gomez and Iuvone, 1998; Gastel et al., 1998; Greve et al., 1999; Chen and Baler, 2000).

In the present study we found that a cAMP analog, which activates PKA, elevates melatonin levels at all the times of the day (Fig. 2), even in the morning when darkness is ineffective. This observation indicates that the mechanisms that gate darknessstimulated melatonin synthesis in the early part of the day must be located upstream of cAMP. The observation that forskolin treatment also failed to induce melatonin synthesis early in the day (Fig. 3) suggests that calmodulin-stimulated adenylyl cyclase activity may be low in the morning and a limiting factor in darkevoked melatonin biosynthesis.

The results of the experiment shown in Figure 4 demonstrate that cyclic nucleotide phosphodiesterase (PDE) activity is not involved in this phenomenon because IBMX (a specific PDE inhibitor) alone or in combination with forskolin does affect melatonin synthesis at ZT 0-6 or ZT 3-9 (Fig. 4). This result indicates that changes of cAMP synthesis rather than breakdown are important for gating melatonin synthesis at ZT $0-6$ or ZT 3-9. It must mentioned, however, that forskolin and IBMX may act synergistically to potentiate the effect of cAMP analog and forskolin at least at ZT 6-12 and ZT 9-12 (Fig. 4), and such a result is in agreement with previous experimental observations (Gan et al., 1995).

Gating by the circadian clock in retina has been observed previously in the retina (Iuvone et al., 1999) and in the SCN (Ginty et al., 1993; Obrietan et al., 1999), but these studies identified neither the location of the gate nor the mechanisms by which the circadian clock controls the gate. The present investigation demonstrates that cAMP levels and AC1 mRNA abundance show a clear circadian rhythm in retina (Fig. 5) and photoreceptor cells (Fig. 6). These results are consistent with studies showing circadian control of cAMP levels in chick photoreceptors and pinealocytes (Nikaido and Takahashi, 1998; Ivanova and Iuvone, 2003) and of phospho-cAMP response element-binding protein levels in Xenopus photoreceptors (Liu and Green, 2002), all of which are high at night.

A previous study has reported that in the rat retina $A C 1$ mRNA is expressed predominantly in the photoreceptors (Xia et al., 1993), whereas a recent immunohistochemical study failed to detect AC1 protein (or other calcium-sensitive adenylyl cyclase) in the photoreceptors (Abdel-Majid et al., 2002). Our results (Fig. $7 A, B, D, E)$ demonstrate that $\mathrm{AC} 1$ protein is expressed in the photoreceptors, but it is detectable only during the night (Fig. $7 \mathrm{D}, E)$. The fact that $\mathrm{AC} 1$ protein levels are low during the day and high at night further supports our conclusion that melatonin synthesis cannot be induced by darkness and forskolin at ZT 0 and ZT 3 because cAMP synthesis is insufficient at these times.

The transcription of the clock-controlled gene can be activated by the circadian transcription factors BMAL1 and CLOCK, if the genes contain E-boxes in their promoters (Jin et al., 1999;
Chong et al., 2000; Ripperger et al., 2000), as do the Aa-nat and $A C 1$ promoters (Chen and Baler, 2000; Chan et al., 2001). Our study demonstrated that $A C 1$ promoter activity is directly regulated by the clock via the action of the BMAL1:CLOCK complex and contributes to the circadian gating of melatonin synthesis in photoreceptor cells (Fig. 8). Similarly, it was reported that $\mathrm{Ca}^{2+}$ / calmodulin-stimulated adenylyl cyclase activity is regulated by a circadian clock in chicken pineal photoreceptors (Nikaido and Takahashi, 1998).

The circadian rhythm of cAMP in retina, with a trough early in the day when neither darkness nor forskolin stimulates melatonin synthesis, strongly suggests that regulation of the cAMP level represents a gate controlling melatonin biosynthesis. The circadian expression of $A C 1 \mathrm{mRNA}$ and protein in photoreceptors indicates that the cyclase contributes to gating of melatonin synthesis by the clock; however, it does not exclude the involvement of other $\mathrm{Ca}^{2+} /$ calmodulin-stimulated or $\mathrm{Ca}^{2+}$-independent adenylyl cyclases or of cyclic nucleotide phosphodiesterases in the gating mechanism. These await further investigation.

A potential flaw in our hypothesis is that the $A a$-nat and $A C 1$ transcript rhythms are not in phase. $A C 1$ mRNA peaks during the daytime, whereas Aa-nat transcript levels are highest at night. If rhythmic transcription is driven primarily by BMAL1:CLOCKmediated transactivation, the rhythms of expression of both genes might be expected to be synchronous; however, this temporal discrepancy may be attributable to the presence of other regulatory inputs to the two promoters. In particular, cAMP has opposite effects on the $A C 1$ and $A a$-nat promoters. cAMP inhibits $A C 1$ promoter activity (Chan et al., 2001) but stimulates the Aa-nat promoter (Baler et al., 1997). The low level of cAMP during the daytime may be permissive for BMAL1:CLOCKmediated stimulation of $A C 1$ transcription, leading to peak levels of $A C 1$ mRNA during the daytime. Conversely, the high level of cAMP at night may enhance BMAL1:CLOCK-mediated Aa-nat transcription, which is highest at night.

Although cAMP does not appear to be directly involved in visual transduction, it regulates several aspects of photoreceptor metabolism linked to light and dark adaptation. For example, cAMP is involved in the regulation of membrane turnover in rod outer segments, cone retinomotor movement, the activity of the $\mathrm{G}_{\beta} \gamma$-binding protein phosducin, and melatonin biosynthesis, as well as some types of retinal degeneration and photoreceptor apoptosis (Besharse et al., 1982; Yoshida et al., 1994; Weiss et al., 1995; Fassina et al., 1997; Alfinito and Townes-Anderson, 2002). The fact that the retinal circadian clock controls the cAMP signaling cascade indicates that this clock has a more general and profound impact on retinal functions than previously thought. In particular, it is important to note that the circadian clock gates the cAMP signaling cascade at the night-to-day transition when the photoreceptors need to rapidly adapt to changes in the levels of illumination.

In mammals, the central circadian pacemaker controlling the vast majority of circadian rhythms, including that of pineal melatonin biosynthesis, is located within the SCN of the hypothalamus (Klein et al., 1991). CRE-mediated gene expression in the SCN shows circadian rhythmicity, and photic stimulation increases CRE-mediated transcription during the subjective night but not the subjective day (Obrietan et al., 1999). Our results (Fig. 9) suggest that the circadian clock may gate the cAMP signaling cascade in the SCN, as it does in the retina and pineal gland. Thus, gating of cAMP signaling may play a key role in input and output components of the central circadian axis, the retina, the SCN, and the pineal gland. 
In conclusion, our results demonstrated that (1) melatonin synthesis in the retina is gated by the retinal circadian clock, (2) the gate appears to be located at the level of adenylyl cyclase activity, (3) AC1 transcription, protein, and cAMP levels show clear circadian rhythms, (4) AC1 promoter activity is controlled by the circadian clock through an E-box-mediated action of the BMAL1:CLOCK complex, and, finally, (5) AC1 expression is similarly regulated in the SCN.

\section{References}

Abdel-Majid RM, Tremblay F, Baldrigde WH (2002) Localization of adenylyl cyclase proteins in rodent retina. Mol Brain Res 101:62-70.

Alfinito PD, Townes-Anderson E (2002) Activation of mislocalized opsin kills rod cells: a novel mechanism for rod cell death in retinal disease. Proc Natl Acad Sci USA 99:5655-5660.

Alonso-Gomez AL, Iuvone PM (1998) Melatonin biosynthesis in cultured chick retinal photoreceptor cells: calcium and cyclic AMP protect seroto$\operatorname{nin} \mathrm{N}$-acetyltransferase from inactivation in cycloheximide-treated cells. J Neurochem 65:1054-1060.

Anderson FE, Green CB (2000) Symphony of rhythms in Xenopus laevis retina. Microsc Res Tech 50:360-372.

Avendano G, Butler BJ, Iuvone PM (1990) $\mathrm{K}^{+}$-evoked depolarization induces serotonin $\mathrm{N}$-acetyltransferase activity in photoreceptor-enriched retinal cell cultures. Involvement of calcium influx through L-type channels. Neurochem Int 17:117-126.

Baler R, Covington S, Klein DC (1997) The rat arylalkylamine $N$-acetyltransfersae gene promoter. J Biol Chem 272:6979-6985.

Bernard M, Iuvone PM, Cassone VM, Roseboom PH, Coon SL, Klein DC (1997) Avian melatonin synthesis: photic and circadian regulation of serotonin $\mathrm{N}$-acetyltransferase mRNA in the chicken pineal gland and retina. J Neurochem 68:213-224.

Besharse JC, Iuvone PM (1983) Circadian clock in Xenopus eye controlling retinal serotonin $N$-acetyltransferase. Nature 305:133-135.

Besharse JC, Dunis DA, Burnside B (1982) Effects of cyclic adenosine 3', 5' monophosphate on photoreceptor disc shedding and retinomotor movement. Inhibition of rod shedding and stimulation of cone elongation. J Gen Physiol 79:775-790.

Cahill GM, Besharse JC (1993) Circadian clock functions localized in Xenopus retinal photoreceptors. Neuron 10:573-577.

Cahill GM, Besharse JC (1995) Circadian rhythmicity in vertebrates retina: regulation by a photoreceptor oscillator. Prog Retin Eye Res 14:268-291.

Chan GC, Lernmark U, Xia Z, Storm DR (2001) DNA elements of the type 1 adenylyl cyclase gene locus enhance reporter gene expression in neurons and pinealocytes. Eur J Neurosci 13:2054-2066.

Chen W, Baler R (2000) The rat arylalkylamine $N$-acetyltransferase E-box: different use in a master vs. slave oscillator. Mol Brain Res 81:43-50.

Chong NW, Bernard M, Klein DC (2000) Characterization of the chicken serotonin $\mathrm{N}$-acetyltransferase gene. Activation via clock gene heterodimer/E box interaction. J Biol Chem 275:32991-32998.

Faillace MP, Sarmiento MI, Siri LN, Rosenstein RE (1994) Diurnal variations in cyclic AMP and melatonin content of golden hamster retina. J Neurochem 62:1995-2000.

Fassina G, Aluigi MG, Gentleman S, Wong P, Chai T, Albini A, Noonan DM (1997) The cAMP analog 8-Cl-cAMP inhibits growth and induces differentiation and apoptosis in retinoblastoma cells. Int J Cancer 72:1088-1094.

Fukuhara C, Dirden JC, Tosini G (2001) Photic regulation of melatonin in rat retina and the role of proteasomal proteolysis. NeuroReport 12:3833-3837.

Fukuhara C, Dirden JC, Tosini G (2002) Regulation of Period1 expression in cultured rat pineal. NeuroSignals 11:103-114.

Gan J, Alonso-Gomez AL, Avendano G, Johnson B, Iuvone PM (1995) Melatonin biosynthesis in photoreceptor-enriched chick retinal cell cultures: role of cyclic AMP in the $\mathrm{K}(+)$-evoked, $\mathrm{Ca}(2+)$-dependent induction of serotonin $N$-acetyltransferase activity. Neurochem Int 27:147-155.

Gastel JA, Roseboom PH, Rinaldi PA, Weller JL, Klein DC (1998) Melatonin production: proteasomal proteolysis in serotonin $\mathrm{N}$-acetyltransferase regulation. Science 279:1358-1360.

Ginty DD, Kornhauser JM, Thompson MA, Bading H, Mayo KE, Takahashi JS (1993) Regulation of CREB phosphorylation in the suprachiasmatic nucleus by light and a circadian clock. Science 260:238-241.
Greve P, Alonso-Gomez AL, Bernard M, Ma M, Haque R, Klein DC, Iuvone PM (1999) Serotonin $N$-acetyltransferase mRNA levels in photoreceptorenriched chicken retinal cell cultures: elevation by cyclic AMP. J Neurochem 73:1894-1900.

Hamm HE, Takahashi JS, Menaker M (1983) Light-induced decrease of serotonin $\mathrm{N}$-acetyltransferase activity and melatonin in the chicken pineal gland and retina. Brain Res 266:287-293.

Hasegawa M, Cahill GM (2000) Modulation of rhythmic melatonin synthesis in Xenopus retinal photoreceptors by cyclic AMP. Brain Res 824:161-167.

Iuvone PM (1995) Cell biology and metabolic activity of photoreceptor cells: light-evoked and circadian regulation. In: Neurobiology and clinical aspects of the outer retina (Djamgoz MBA, Archer S, Vallerga S, eds), pp 25-55. London: Chapman and Hall.

Iuvone PM, Besharse JC (1983) Regulation of indolamine $N$-acetyltransferase activity in the retina: effects of light and dark, protein synthesis inhibitors and cyclic nucleotide analogs. Brain Res 273:111-119.

Iuvone PM, Besharse JC (1986a) Cyclic AMP stimulates serotonin $\mathrm{N}$-acetyltransferase activity in Xenopus retina in vitro. J Neurochem 46: 33-39.

Iuvone PM, Besharse JC (1986b) Involvement of calcium in the regulation of serotonin $N$-acetyltransferase in retina. J Neurochem 46:82-88.

Iuvone PM, Avendano G, Butler BJ, Adler R (1990) Cyclic AMP-dependent induction of serotonin $\mathrm{N}$-acetyltransferase activity in photoreceptorenriched chick retinal cell cultures: characterization and inhibition by dopamine. J Neurochem 55:673-682.

Iuvone PM, J Gan, Avendano G (1991) K(+)-evoked depolarization stimulates cyclic AMP accumulation in photoreceptor-enriched retinal cell cultures: role of calcium influx through dihydropyridine-sensitive calcium channels. J Neurochem 57:615-621.

Iuvone PM, Bernard M, Alonso-Gomes A, Greve P, Cassone VM, Klein DC (1997) Cellular and molecular regulation of serotonin $\mathrm{N}$-acetyltransferase activity in chicken retinal photoreceptors. Biol Signals 6:217-224.

Iuvone PM, Chong NW, Bernard M, Brown AD, Thomas KB, Klein DC (1999) Melatonin biosynthesis in chicken retina. Regulation of tryptophan hydroxylase and arylalkylamine $N$-acetyltransferase. Adv Exp Med Biol 460:31-41.

Iuvone PM, Brown AD, Haque R, Weller JB, Zawilska JB, Chaurasia SS, Ma M, Klein DC (2002) Retinal melatonin production: role of proteasomal proteolysis in circadian and photic control of arylalkylamine $\mathrm{N}$-acetyltransferase. Invest Ophthalmol Vis Sci 43:564-572.

Ivanova TN, Iuvone PM (2003) Circadian rhythm and photic control of cAMP level in chick retinal cell cultures: a mechanism for coupling the circadian oscillator to the melatonin-synthesizing enzyme, arylalkylamine $N$-acetyltransferase, in photoreceptor cells. Brain Res 991:96-103.

Jin X, Shearman LP, Weaver DR, Zylka MJ, De Vries GJ, Reppert SM (1999) A molecular mechanism regulating rhythmic output from the suprachiasmatic circadian clock. Cell 96:57-60.

Klein DC, Moore RY, Reppert SM (1991) Suprachiasmatic nucleus: the mind's clock. New York: Oxford UP.

Klein DC, Coon SL, Roseboom PH, Weller JL, Bernard M, Gastel JA, Zazt M, Iuvone PM, Rodriguez IR, Begay V, Falcon J, Cahill GM, Cassone VM, Baler R (1997) The melatonin rhythm-generating enzyme: molecular regulation of serotonin $\mathrm{N}$-acetyltransferase in the pineal gland. Recent Prog Horm Res 52:307-358.

Klein DC, Ganguly S, Coon S, Weller JL, Obsil T, Hickman A, Dyda F (2002) 14-3-3 Proteins and photoneuroendocrine transduction: role in controlling the daily rhythm in melatonin. Biochem Soc Trans 30:365-373.

Liu C, Fukuhara C, Wessel III JH' Iuvone PM, Tosini G (2004) Localization of Aa-nat mRNA in the rat retina by fluorescence in situ hybridization and laser capture microdissection. Cell Tissue Res 315:197-201.

Liu X, Green CB (2002) Circadian regulation of nocturnin transcription by phosphorylated CREB in Xenopus retinal photoreceptor cells. Mol Cell Biol 22:7501-7511.

Nikaido SS, Takahashi JS (1998) Day/night differences in the stimulation of adenylate cyclase activity by calcium/calmodulin in chick pineal cell cultures: evidence for circadian regulation of cyclic AMP. J Biol Rhythms 13:479-493.

Niki T, Hamada T, Ohtomi M, Sakamoto K, Suzuki S, Kako K, Hosoya Y, Horikawa K, Ishida N (1998) The localization of the site of arylalkylamine $\mathrm{N}$-acetyltransferase circadian expression in the photoreceptor cells of mammalian retina. Biochem Biophys Res Commun 248:115-120. Obrietan K, Impey S, Smith D, Athos J, Storm DR (1999) Circadian regula- 
tion of cAMP response element-mediated gene expression in the suprachiasmatic nuclei. J Biol Chem 274:17748-17756.

Pierce ME, Sheshberadaran H, Zhang Z, Fox LE, Applebury ML, Takahashi JS (1993) Circadian regulation of iodopsin gene expression in embryonic photoreceptors in retinal cell culture. Neuron 10:579-584.

Redburn DA, Mitchell CK (1989) Darkness stimulates rapid synthesis and release of melatonin in rat retina. Vis Neurosci 3:391-403.

Ripperger JA, Shearman LP, Reppert SM, Schibler U (2000) CLOCK, an essential pacemaker component, controls expression of the circadian transcription factor DBP. Genes Dev 14:679-689.

Rollag M, Niswender GD (1976) Radioimmunoasssay of serum concentrations of melatonin in sheep exposed to different lighting regimens. Endocrinology 98:482-489.

Sakamoto K, Ishida N (1998) Circadian expression of serotonin $\mathrm{N}$-acetyltransferase mRNA in the rat retina. Neurosci Lett 245:113-116.

Sakamoto K, Oishi K, Ishida N (2002) Ontogeny of circadian expression of serotonin $\mathrm{N}$-acetyltransferase mRNA in the rat retina. Neurosci Lett 317:53-55.

Tosini G, Fukuhara C (2002) The mammalian retina as a clock. Cell Tissue Res 309:119-126.

Tosini G, Menaker M (1996) Circadian rhythms in cultured mammalian retina. Science 272:419-421.
Tosini G, Menaker M (1998) The clock in the mouse retina: melatonin synthesis and photoreceptor degeneration. Brain Res 789:221-228.

Tzavara E-Th, Pouille Y, Defer N, Hanoune J (1996) Diurnal variation of the adenylyl cyclase type 1 in the rat pineal gland. Proc Natl Acad Sci USA 93:11208-11212.

Uchida K, Iuvone PM (1999) Intracellular $\mathrm{Ca}^{2+}$ concentrations in cultured chicken photoreceptor cells: sustained elevation in depolarized cells and the role of dihydropyridine-sensitive $\mathrm{Ca}^{2+}$ channels. Mol Vis 5:1.

Weiss ER, Hao Y, Dickerson CD, Osawa S, Shi W, Zhang L, Wong F (1995) Altered cAMP levels in retinas from transgenic mice expressing a rhodopsin mutant. Biochem Biophys Res Commun 216:755-761.

Werblin FS, Dowling JE (1969) Organization of the retina of the mudpuppy, Necturus maculosus. II. Intracellular recording. J Neurophysiol 32:339-355.

Xia Z, Storm DR (1997) Calmodulin-regulated adenylyl cyclases and neuromodulation. Curr Opin Neurobiol 7:391-396.

Xia Z, Choi E-J, Fan W, Blazynski C, Storm DR (1993) Type I calmodulinsensitive adenylyl cyclase is neural specific. J Neurochem 60:305-311.

Yoshida T, Willardson B, Wilkins JF, Jensen GJ, Thornton B, Bitensky MW (1994) The phosphorylation state of phosducin determines its ability to block transducin subunit interactions and inhibit transducin binding to activated rhodopsin. J Biol Chem 269:24050-24057. 